\title{
Relación del Bienestar Psicológico, Subjetivo y Laboral. Análisis en profesionales de la salud en Ecuador
}

\section{Relationship of Psychological, Subjective and Labor Well-being. Analysis in health professionals from Ecuador}

\author{
Yánez-Ramos, Dayana ${ }^{1}$ y Moreta-Herrera, Carlos Rodrigo ${ }^{1}$
}

\begin{abstract}
Resumen:
Objetivo: Conocer la predictibilidad del Bienestar Psicológico y Subjetivo en el Bienestar Laboral en una muestra de profesionales sanitarios del Ecuador. Método: Estudio descriptivo correlacional, predictor y de corte transversal con pruebas de evaluación del bienestar. Muestra: 144 trabajadores $(65,3 \%$ mujeres), entre 23 a 59 años $(M=33,6$; Ds= 8,2). Procedentes de tres unidades de salud de la provincia de Bolívar, Ecuador. Un 47,2\% son médicos y el 53,8\% restante son personal de enfermería. Resultados: Los niveles de Bienestar Psicológico, Subjetivo y Laboral son adecuados. Se constató correlaciones moderadas y bajas del Bienestar Psicológico y Subjetivo con el Bienestar Laboral. El 22\% de la varianza del Bienestar Laboral es predicho por el Bienestar Psicológico y la Satisfacción de la vida. Conclusión: El Bienestar Psicológico y Subjetivo, predicen el Bienestar Laboral en profesionales de la salud.
\end{abstract}

Palabras Clave: Bienestar, afectividad, satisfacción, salud, trabajo.

\begin{abstract}
:
Objective: To know the predictability of Psychological and Subjective Wellbeing in Labor Welfare in a sample of health professionals in Ecuador. Method: Descriptive correlational, predictive and cross-sectional study with welfare assessment tests. Sample: 144 workers $(65.3 \%$ women $)$, between 23 and 59 years $(\mathrm{M}=33.6$; $\mathrm{Ds}=8.2)$. Coming from three health units in the province of Bolívar, Ecuador. $47.2 \%$ are doctors and the remaining 53.8\% are nurses. Results: The levels of Psychological, Subjective and Labor Welfare are adequate. Moderate and low correlations of Psychological and Subjective Well-being in Labor Welfare were found. 22\% of the variance of Labor Welfare is predicted by Psychological Welfare and Life Satisfaction. Conclusion: Psychological and Subjective Well-being, predict Labor Well-being in health professionals.
\end{abstract}

Keywords: Well-being, afectivity, satisfaction, health, job.

\footnotetext{
${ }^{1}$ Pontificia Universidad Católica del Ecuador Sede Ambato
}

*Correspondencia: rmoreta@pucesa.edu.ec 
Las condiciones laborales que se les da al personal del sector de la salud, así como de otros sectores sociales, pueden entrar en algún grado y tipo de conflicto porque muchas de estas se deciden en base a los aspectos económicos, financieros y políticos tanto nacional como internacional, sin considerar el elemento humano y personal. Con lo que se provoca un cambio en la dinámica laboral que incide en el bienestar y la salud de los trabajadores (Crespo, 2014). La importancia que tienen estos elementos guarda relación tanto con las tareas de cuidado y atención al usuario (Fierro-Arias, Simón-Díaz, Ponce-Olivera \& Arenas-Guzmán, 2018); como con las de exposición a riesgos y vulnerabilidad (Paris, 2016; García, Cova-Solar, Bustos-Torres \& Reyes-Pérez, 2018).

El analizar el bienestar en el trabajo contribuye a darle desde la investigación una mirada positiva a la actividad laboral. Principalmente, porque los estudios sobre la psicología del trabajo no deben centrarse únicamente en la sintomatología o los problemas relacionados (Organización Mundial de la Salud [OMS], 2013), sino también en la calidad de vida (Diener, Oishi \& Lucas, 2003; Blanch, Sahagún, Cantera, \& Cervantes, 2010), la salud general (Oramas, Santana \& Vergara, 2006; Moreta-Herrera et al., 2018), la cotidianidad (Paschoal, Álvaro \& Barreiros -Porto, 2015) y especialmente en la cultura organizacional y la productividad (Oswald, Proto \& Sgroi, 2015; Peña-Garzón \& Torrente-Rocha, 2020).

En cuanto a explicar el bienestar en el trabajo, se evidencia un discurso preferencial por los modelos explicativos personalistas y patológicos; y descuidando los aspectos contextuales y organizacionales. Estos modelos suelen considerar pasivamente al individuo, como un mero receptor de los movimientos organizacionales y sus efectos. Y es por ello por lo que, usualmente se estudia el malestar laboral, la sintomatología y su psicopatología (Blanch et al., 2010), las experiencias negativas del trabajo como distrés, burnout, riesgos laborales (García et al., 2018) entre otros. Al punto de que estos estudios sobrepasan a los que tratan acerca de las experiencias laborales positivas (Schaufeli \& Bakker, 2004). Esta tendencia se explica al parecer por la mirada 'clínica' que se les da a los fenómenos psicológicos sociales y deforma la interpretación de la experiencia laboral.

Si bien es cierto que las investigativas psicológicas actuales sobre el abordaje positivo de la experiencia laboral en especial en personal de salud (médicos, enfermeras y otros) es limitada, incompleta y muchas veces sesgada, queda claro que las organizaciones no solo son generadoras de problemas en el bienestar y calidad de vida de las personas, sino también son promotoras de salud y de cuidados personales (Nader, Bernate \& SantaBárbara, 2014) y por ello su importancia.

\section{Bienestar individual y bienestar laboral}

El bienestar no solo son los aspectos que ayudan a 'sentirse bien', o por el que se debería serlo en las personas; sino también la percepción y valoración que se construyen sobre la realidad y que genera satisfacción y felicidad a partir de la cotidianidad y lo 'positivo' de la vida (Seligman, 2003); aunque esa construcción de la realidad podría ser sesgada e inclinarse para favorecer los niveles de bienestar (Torres-Salazar, Moreta-Herrera, RamosRamírez, \& López-Castro, 2020) como un mecanismo propio de cuidado de la esfera psíquica.

Desde lo perceptual, al bienestar se lo relaciona con lo subjetivo (hedónico), que es la presencia significativa de emociones y sen- 
timientos positivos (Watson, 1988; Medrano et al., 2015); junto con la satisfacción que le genera la vida globalmente (Diener et al., 1985; Padrós, Gutiérrez \& Medina, 2015). Y también con el esfuerzo y el interés del individuo en autosuperarse (eudaimónico) al proponerse objetivos y alcanzarlos (Ryff, 1989; Díaz, Stavraki, Blanco \& Gandarillas, 2015). Estos elementos perceptuales (hedónico y eudaimónico) son necesarios e interdependientes para su funcionamiento. Pues el ser feliz y estar satisfecho no basta para alcanzar el bienestar pleno, sino que se requiere de mecanismos de interpretación y valoración de la experiencia vivida para que resulte como satisfactoria, agradable o positiva (Diener, Oishi \& Lucas, 2003).

La percepción del bienestar individual predice el interés, el apoyo, la preocupación social y la salud mental (Moreta, Gaibor \& Barrera, 2017; Moreta-Herrera et al., 2018), la resiliencia y el afrontamiento (Arrogante, Pérez-García, \& Aparicio-Zaldívar, 2015) entre otros; todos sobre aspectos generales de la vida, pero también en variables laborales como la satisfacción y la felicidad en el trabajo (Van Aerden, Moors, Levecque \& Vanroelen, 2015; Fierro-Arias et al., 2018). Sin embargo, no establece con precisión la relación causal con otras variables; por ejemplo, se conoce de trabajadores que con elevada productividad laboral evidencian baja felicidad o satisfacción (Peiró et al., 2014). Esto porque el trabajo es solo un aspecto dentro de un abanico de actividades del individuo y no necesariamente guarda armonía plena con las actividades restantes. De esta manera, una valoración generalista y personalista del bienestar sin considerar los elementos del trabajo puede ser insuficiente.

Así, es necesario profundizar en el análisis del bienestar desde las particularidades propias del trabajo, que se identifica como
Bienestar Laboral (BL). El cual se entiende como el grado óptimo de satisfacción del trabajador; correspondiente a un estado mental y conductual dinámico y determinado por las necesidades y las expectativas del trabajador (Restrepo Escobar \& López Velásquez, 2013). Se concibe como un constructo multifactorial (biopsicosocial) (OIT, 2016) y estructural que es parte de la calidad de vida laboral y responde a la evaluación positiva subjetiva del trabajo que le permite sentirse satisfecho por medios de sus percepciones y valoraciones (González \& Bousoño, 1996).

El BL según el modelo de Blanch y otros (2010), se constituye por los aspectos emocionales, las expectativas y las competencias del trabajador (Bienestar psicosocial) por una parte; y la respuesta somática, el desgaste y la alienación por el trabajo (efectos colaterales) por otra. De esta forma el BL es inclusivo, pues, integra el entorno físico, los riesgos, la organización laboral, las tareas, las relaciones, la salud personal, la capacidad e incluso el estrés familiar (Suomaa, Yrjänheikki, Savolainen \& Jokiluoma, 2011). Pues, se centra en los atributos de los colaboradores de una organización, en lugar de considerar únicamente el entorno, el papel regulatorio del trabajo u otras influencias que afecten el bienestar (Ravenswood, 2011).

Además, esta mirada no es personalista, sino que responde también a la satisfacción de las necesidades organizacionales (EstradaRodríguez \& Ramírez-Reyes, 2010). Este no recae explícitamente en si la percepción que se tiene del sentir del trabajo, lo valora como bueno o no; sino también en la estimación del contexto laboral para proporcionar los recursos organizacionales adecuados para sentir un adecuado bienestar laboral. Así, el BL es una consecuencia, reacción o efecto del aporte organizacional como pueden ser por ejemplo las condiciones de trabajo o los cambios labo- 
rales (De Cuyper, Isaksson \& De Witte, 2005).

El BL en la práctica, es sensible a las condiciones de trabajo que requieren especificaciones mínimas para su despliegue óptimo (Florencia, 2015; Restrepo Escobar \& López Velásquez, 2013). Se correlaciona con variables laborales como la satisfacción o el agotamiento (Landa, Berrios-Martos, López-Zafra \& Aguilar Luzón, 2006; Van Aerden, Moors, Levecque, \& Vanroelen, 2015; Fierro-Arias et al., 2018), el rendimiento laboral (Peiró et al., 2014) y el clima organizacional (GálvezRuiz et al., 2017). Por lo que su recurso explicativo es bastante pertinente en la investigación organizacional, en especial en el personal de asistencia y salud (Crespo, 2014; Arrogante, Pérez-García \& Aparicio-Zaldívar, 2015; Donoso, Demerouti, \& Jiménez, 2015; Paris, 2016; Gaibor-González \& MoretaHerrera, 2020).

Tanto la percepción personal del bienestar en general (subjetivo y psicológico) como el BL hoy en día, se los estudia como constructos independientes, dado que no existe evidencia aún de que formen parte de un mismo constructo. Por lo que la interacción de estos dos elementos entre sí puede ser motivo de análisis y estudio. De todos modos, aún existen limitantes en el Ecuador y en la región en general acerca de los aspectos individuales y organizacionales que encierran el bienestar. Si bien existen estudios que relacionan el Bienestar Psicológico y el Subjetivo con el Laboral (Requena, 2000; Garrosa, Moreno, Boada \& Blanco, 2010; Romeiro Martínez, 2015), estos no están plenamente esclarecidos y sin estudios confirmatorios sostenidos en el tiempo; también no se presentan estudios en muestras particulares como en el personal de salud por ejemplo, por lo que el avance sobre esta línea aún tiene interrogantes e inquietudes que despejar.
Por tal razón y para el presente estudio se considera necesario abordar los siguientes objetivos e hipótesisi de trabajo: a) Conocer la relación existente del Bienestar Psicológico y Subjetivo en el Bienestar Laboral en una muestra de profesionales de la salud del Ecuador; como H1 se considera que esta relación es existente de manera significativa la relación entre estas variables; y b) Determinar el nivel predictor del Bienestar Psicológico y Subjetivo en el BL; en la H2, se estima que el BP y el BS influyen en el cambio de la varianza del $\mathrm{BL}$.

\section{Método}

El presente trabajo comprende un estudio de carácter cuantitativo y descriptivo, de carácter correlacional, predictor y de corte transversal (Ato, López, \& Benavente, 2013) entre el Bienestar Psicológico y Subjetivo con el Bienestar Laboral en una muestra de profesionales de la salud (personal médico y de enfermería) que laboran en centros hospitalarios de la provincia de Bolívar, Ecuador.

\section{Participantes}

El estudio estuvo compuesto por 144 trabajadores de la salud. Un 34,7\% son hombres y un $65,3 \%$ mujeres. Las edades fluctuaron entre 23 a 59 años $(M=33,6$; $D s=8,2)$. Además, un $86,1 \%$ se autoidentifica étnicamente como mestizos(as) y un 13,9\% como indígenas.

Los participantes son trabajadores de tres unidades de salud: Hospital "A" (38,9\%), Centro de Salud "Ch" (39,6\%) y Hospital "B" $(21,5 \%)$ pertenecientes a la provincia de Bolívar en Ecuador. Un 47,2\% está compuesto por médicos(as), mientras que el $53,8 \%$ restante son personal de enfermería (enfermeras y auxiliares). La antigüedad promedio de los trabajadores es de $\mathrm{M}=5,8$ años; $\mathrm{Ds}=6,1$ y con una remuneración mensual de $\mathrm{M}=1082,39 \mathrm{USD} ; \mathrm{Ds}=321,05$. 
La selección de los participantes se realizó a través de un muestreo no probabilístico con criterios de inclusión que fueron: a) Ser profesional de la salud (médico, enfermera o auxiliar); b) Ser trabajador regular en uno de las tres unidades de salud; y c) Carta de consentimiento firmada para participar en el estudio.

\section{Instrumentos}

Escala de Bienestar Psicológico (Ryff, 1989) en la versión de van Dierendonck (2004) adaptado al castellano por Díaz y otros (2006). El cual cuenta con 39 ítems en una escala de respuesta Likert de seis opciones (Entre muy de acuerdo [6] y muy en desacuerdo [1]). Los factores encargados de valorar fueron: Autoaceptación, Relaciones Positivas, Autonomía, Dominio del Entorno, Crecimiento Personal y Propósito de vida. Datos preliminares en población ecuatoriana muestran una consistencia interna de $\alpha=, 89$ equivalente a elevada (Moreta, Gaibor \& Barrera, 2017). Mientras que en el presente estudio los resultados fueron de $\alpha=, 9$.

Escala de Satisfacción con la vida (Diener, Emmons, Larsen \& Griffin, 1985) en la versión traducida al castellano y adaptada en población mexicana (Padrós, Gutiérrez \& Medina, 2015). Que se encarga de realizar una apreciación cognitiva del Bienestar Subjetivo desde la valoración de la satisfacción general que mantiene con su vida el participante a través de un cuestionario de cinco preguntas con opciones de respuesta de siete niveles (de totalmente de acuerdo [7] a totalmente desacuerdo [1]), en el que los valores altos reflejan mayor satisfacción y viceversa. La fiabilidad de la prueba muestra que es alta en población ecuatoriana adulta con valores de $\alpha=, 81$ (Arias \& García, 2018). Para el presente estudio aplicado a profesionales de la salud la consistencia interna encontrada fue de $\alpha=, 73$ equivalente a fiabilidad aceptable.

Escala de Afecto positivo y negativo (PANAS, Watson, 1988) Diseñado para la evaluación y presencia de los afectos postivos (AP) y negativos (AN). El instrumento cuenta con 20 ítems que se responden con una escala Likert de cinco opciones (1) levente a (5) extremadamente. La versión de la prueba traducida al castellano corresponde a lo propuesto por Joiner y otros (1997). Estudios previos de validación en población de la región señalan la existencia de una fiabilidad moderadamente alta de $\alpha=, 82$ para la prueba (Medrano et al., 2015). En la muestra de análisis, se encontró valores de $\alpha=, 73$ para afecto positivo y $\alpha=, 68$ para afecto negativo; estas consistencias internas muestran una fiabilidad aceptable para la investigación.

\section{Escala de Bienestar Laboral General} (Blanch et al., 2010). Para evaluar la percepción de bienestar con respecto a su trabajo en función de dos factores: a) Bienestar Psicosocial con 42 ítems que valora los afectos, competencias y expectativas; y b) Efectos colaterales que mide somatización, alineamiento y agotamiento con 13 ítems. La respuesta a los ítems en la escala de Bienestar Psicosocial comprendió respuestas a través de un diferencial semántico (Insatisfacción - Satisfacción); mientras que en la escala de Efectos Colaterales se usó una escala Likert de 7 opciones (Nunca - Siempre).

En cuando a las propiedades psicométricas, se encontró valores elevados de consistencia interna en población española con $\alpha=$,97 para Bienestar Psicosocial y $\alpha=, 91$ para Efectos Colaterales. Mientras que en el presente estudio con ecuatorianos los valores fueron de $\alpha=, 97$ para Bienestar Psicosocial y $\alpha=, 73$ para Efectos Colaterales. 


\section{Procedimiento}

El equipo de investigación tras la correspondiente autorización de las casas de salud realizó el proceso de evaluación al personal de salud, quienes previamente fueron informados sobre el proyecto y los objetivos del mismo y dieron su consentimiento por escrito. El tiempo de duración de las pruebas fue de aproximadamente 30 minutos, que se realizó de forma individual en sus lugares y espacios de trabajo.

Posteriormente con las evaluaciones, se procedió a su depuración y sistematización en bases de datos electrónicas para los análisis estadísticos respectivos, la verificación de hipótesis y la formulación de conclusiones y recomendaciones. El reporte de resultados para su publicación contó con el aval de la comisión de publicaciones de la PUCE Sede Ambato.

\section{Análisis de datos}

El presente trabajo cuenta con tres segmentos de análisis. El primero es un análisis comparativo por grupos de profesiones (personal médico y de enfermería) de los resultados del Bienestar Subjetivo, Psicológico y Laboral por medio de la prueba $t$ of student para muestras independientes $(\mathrm{t})$ y evidenciar o no la presencia de diferencias significativas $(\mathrm{p}<, 05)$; junto a la comparación se analiza el tamaño del efecto del diferencial existente con la prueba de Hedges ajustada $\left(\mathrm{g}\left({ }_{\mathrm{aj}}\right)\right)$.

La segunda parte comprende el análisis correlaciones. Para esta sección se realizan correlaciones generales entre BP, BS y BGL, así como también un análisis del BP y el BS desde los componentes internos del BLG y finalmente entre el BP y el BS entre sí. En todos estos análisis se utilizó el Coeficiente momento-producto de Pearson (r) para conocer la importancia de la fuerza de relación y la significancia $(p<, 05)$ que representa. Final- mente, con los resultados más significativos de este análisis se realizó un Análisis de Regresión Lineal Jerárquico para conocer el potencial predictor que tienen las variables de Bienestar Psicológico y Subjetivo en el BL. El orden de ingreso de variables en el análisis de regresión se lo realizó en función del grado de correlación existente.

La gestión informática de los datos se realizó con el software SPSS en la versión 21 (IBM Corp., 2012).

\section{Resultados}

\section{Análisis de los indicadores de Bienestar} Psicológico y Subjetivo

Los resultados que se presentan en la tabla 1 muestran que los niveles de Satisfacción con la vida, Afecto Positivo y Negativo; y Bienestar Psicológico son altos entre los grupos del personal de salud. En un análisis comparativo se encontró diferencias $(\mathrm{p}<, 05)$ entre los grupos en el factor de Afecto negativo, con mayor presencia entre el personal de enfermería que en el médico. El tamaño del efecto es mediano dado el diferencial existente entre los grupos. En los factores restantes no se presentaron diferencias significativas por lo que se concluye que son invariantes.

\section{Análisis de los indicadores del Bienestar Laboral General}

Como se puede observar en la tabla 2, los análisis descriptivos para los componentes del Bienestar Laboral General son altos. Se encontraron diferencias en el componente de afectos del factor de Bienestar Psicosocial. El grupo del personal de enfermería muestra mayor afectividad que el de los médicos. El tamaño del efecto entre los grupos es moderado debido al diferencial existente. En el resto de los componentes y factores los niveles existentes como tal son invariantes entre los grupos de análisis 
Tabla 1.

Análisis comparativo por grupos del Bienestar Subjetivo y Psicológico

\begin{tabular}{|c|c|c|c|c|c|c|c|}
\hline \multirow{2}{*}{ Variables } & \multicolumn{2}{|c|}{ Personal Médico } & \multicolumn{2}{|c|}{$\begin{array}{l}\text { Personal de } \\
\text { Enfermería }\end{array}$} & \multirow{2}{*}{$F$} & \multicolumn{2}{|c|}{ Contraste } \\
\hline & $M$ & $S$ & $M$ & $S$ & & $t ; p$ & $g_{(a j)}$ \\
\hline Satisfacción Vital & 30.29 & 2.80 & 30.86 & 3.18 & 0.82 & $-1.12 ; .265$ & 0.2 \\
\hline Afecto Positivo & 42.54 & 3.18 & 42.11 & 4.03 & $4.51^{*}$ & $0.73 ; .468$ & 0.1 \\
\hline Afecto Negativo & 13.06 & 2.42 & 13.93 & 2.83 & 2.49 & $-1.98 ; .049$ & 0.3 \\
\hline Bienestar Psicológico & 191.24 & 17.03 & 187.59 & 20.30 & 1.04 & $1.16 ; .248$ & 0.2 \\
\hline
\end{tabular}

Nota: M: Media aritmética; $s$ : Desviación Estándar; F: Prueba de Levene; $t$ : Prueba t pata muestras independientes; $g_{(a j)}:$ Prueba de Hedges ajustada; * $p<.05$

Tabla 2.

Análisis comparativo por grupos del Bienestar Laboral General

\begin{tabular}{|c|c|c|c|c|c|c|c|}
\hline \multirow{2}{*}{ Variables } & \multicolumn{2}{|c|}{ Personal Médico } & \multicolumn{2}{|c|}{$\begin{array}{l}\text { Personal de } \\
\text { Enfermería }\end{array}$} & \multirow{2}{*}{$F$} & \multicolumn{2}{|c|}{ Contraste } \\
\hline & $M$ & $s$ & $M$ & $s$ & & $t ; p$ & $g_{(a j)}$ \\
\hline Afectos & \begin{tabular}{|l|l|}
58.24 \\
\end{tabular} & 6.32 & 61.04 & 8.43 & $5.26^{*}$ & $-2.27 ; .026$ & 0.4 \\
\hline Competencias & 63.56 & 4.56 & 63.91 & 6.42 & 2.19 & $-0.37 ; .710$ & 0.1 \\
\hline Expectativas & 134.25 & 10.84 & 134.95 & 11.82 & 0.09 & $-0.37 ; .714$ & 0.1 \\
\hline Bienestar Psicosocial & 256.04 & 16.76 & 259.89 & 20.38 & 1.69 & $-1.23 ; .221$ & 0.2 \\
\hline Somatización & 29.74 & 3.15 & 29.71 & 3.80 & 1.32 & $0.04 ; .966$ & 0.0 \\
\hline Desgaste & 26.18 & 1.74 & 26.45 & 1.72 & 0.32 & $-0.94 ; .348$ & 0.2 \\
\hline Alienación & 26.18 & 1.74 & 26.45 & 1.72 & 0.32 & $-0.94 ; .348$ & 0.2 \\
\hline Efectos Colaterales & 82.09 & 5.07 & 82.61 & 5.64 & 0.28 & $-0.58 ; .566$ & 0.1 \\
\hline Bienestar Laboral & 338.13 & 19.59 & 342.50 & 23.65 & 1.33 & $-1.20 ; .233$ & 0.2 \\
\hline
\end{tabular}

Nota: M: Media aritmética; s: Desviación Estándar; F: Prueba de Levene; $t$ : Prueba t para muestras independientes; $g_{(a j)}$ : Prueba de Hedges ajustada; * $p<.05$

\section{Correlación del Bienestar Subjetivo, Psico- lógico en el Bienestar Laboral General}

En la tabla 3, en los análisis de correlaciones generales, se observa que el Bienestar Laboral General se correlaciona de forma moderada, positiva y significativa con el Bienestar Psicológico. Así como también de forma baja, positiva y significativa con la Satisfacción con la vida e inversamente con el Afecto Negativo (correlación negativa) del Bienestar Subjetivo.
Así mismo, analizando la dinámica a través de los componentes internos del BLG, el Bienestar Psicosocial (BPS) y Efectos Colaterales (EC) se correlacionan tanto con el Bienestar Psicológico, como con la Satisfacción con la vida y el Afecto Negativo de manera moderada.

Por último, se encontró que los indicadores del Bienestar Psicológico se correlacionan tanto con la Satisfacción con la Vida como con los Afectos Negativos como componentes del Bienestar Subjetivo. 
Tabla 3.

Análisis de correlación del Bienestar Subjetivo y Psicológico en el Laboral

\begin{tabular}{lccccccc}
\hline Variables & $\boldsymbol{E S} \boldsymbol{V}$ & $\boldsymbol{A P}$ & $\boldsymbol{A N}$ & $\boldsymbol{E B P}$ & $\boldsymbol{B P S}$ & $\boldsymbol{E C}$ & $\boldsymbol{B} \boldsymbol{L}$ \\
\hline Satisfacción vital & 1 & $.465^{* *}$ & $-.169^{*}$ & $.473^{* *}$ & $.325^{* *}$ & $.368^{* * *}$ & $.370^{* *}$ \\
Afecto Positivo & & 1 & $-.288^{* *}$ & $.516^{* *}$ & $.211^{*}$ & .152 & $.219^{* *}$ \\
Afecto Negativo & & & 1 & $-.336^{* *}$ & $-.313^{* *}$ & $-.299^{* *}$ & $-.342^{* *}$ \\
Bienestar psicológico & & & & 1 & $.403^{* *}$ & $.353^{* *}$ & $.433^{* *}$ \\
Bienestar Psicosocial & & & & & 1 & $.475^{* *}$ & $.976^{* *}$ \\
Efectos Colaterales & & & & & 1 & $.654^{* *}$ \\
Bienestar Laboral & & & & & & 1
\end{tabular}

Nota: ${ }^{*} p<.05 ;{ }^{* *} p<.01$; ESV: Satisfacción vital; AP: Afecto Positivo; AN: Afecto negativo; EBP: Bienestar Psicológico; BPS: Bienestar Psicosocial; EC: Efectos colaterales; BL: Bienestar Laboral

Tabla 4.

Análisis de predicción del Bienestar Laboral General

\begin{tabular}{|c|c|c|c|c|c|c|}
\hline Modelo & $R_{a}^{2}$ & $F$ & $B$ & $\beta$ & $t$ & VIF \\
\hline Constante & \multirow{3}{*}{, 17} & \multirow{3}{*}{$29,65 * * *$} & 251,97 & & $15,50 * * *$ & \multirow{3}{*}{1,0} \\
\hline Bienestar Psicológico & & &, 47 & ,42 & $5,45 * * *$ & \\
\hline Constante & & & 222,82 & & $12,05 * * *$ & \\
\hline Bienestar Psicológico & \multirow[t]{3}{*}{,22 } & \multirow[t]{3}{*}{$20,55^{* * *}$} & ,33 & ,29 & $3,39 * *$ & 1,31 \\
\hline Satisfacción con la vida & & & 1,85 & 26 & $3,10 * *$ & 1,31 \\
\hline Constante & & & 250,73 & & $10,74 * * *$ & \\
\hline Bienestar Psicológico & \multirow{4}{*}{, 23} & \multirow{3}{*}{$15,20 * * *$} & ,28 & 25 & $2,83 * *$ & 1,40 \\
\hline Satisfacción con la vida & & & 1,78 &, 25 & $3,01 * *$ & 1,32 \\
\hline Afecto negativo & & & $-1,25$ &,- 15 & $-1,93$ & 1,11 \\
\hline Constante & & \multirow{6}{*}{$11,63 * * *$} & & & & \\
\hline Bienestar Psicológico & \multirow{5}{*}{,23 } & & 262,23 & & $9,99^{* * *}$ & \\
\hline Satisfacción con la vida & & & ,31 & ,28 & $2,98^{* *}$ & 1,59 \\
\hline Afecto negativo & & & 1,94 & ,28 & $3,16^{* *}$ & 1,42 \\
\hline \multirow[t]{2}{*}{ Afecto positivo } & & & $-1,36$ &,- 16 & $-2,07^{*}$ & 1,15 \\
\hline & & &,- 51 &,- 09 & $-0,96$ & 1,55 \\
\hline
\end{tabular}

Nota: ${ }^{*} p<, 05 ;{ }^{* *} p<, 01:{ }^{* * *} p<, 001 ;$ Durbin-Watson: 1,$61 ; R_{a}^{2}$ : Coeficiente de determinación ajustado; F: ANOVA; B: Beta no estandarizado; $\beta$ : Beta estandarizado; $t$ : prueba t; VIF: Factor de inflación de la varianza

\section{Análisis de regresión lineal jerárquica}

En la tabla 4, se prueban cuatro modelos construidos a partir de las relaciones planteadas entre las variables de bienestar Psicológico y Subjetivo (Predictoras) y el BL (criterio). El modelo dos señala que efectivamente el Bienestar Psicológico y la Satisfac- ción con la Vida son en conjunto los de mejor predicción del BL. Así lo evidencia el ANOVA $(p<, 001)$ como modelo adecuado y parsimonioso en cada uno de los dos indicadores $(t)$ pues aportan significativamente $(\mathrm{p}<, 05)$ al modelo estimado. 
En conjunto, el modelo empírico puede predecir el 22\% de los cambios de la varianza del Bienestar Laboral General, los coeficientes del modelo responden a los valores de $\beta_{0}=222,82$ como constante; $\beta_{1}=, 33$ para el Bienestar Psicológico y $\beta_{2}=1,85$ para la Satisfacción con la Vida. El factor de varianza inflado es $\mathrm{VIF}<, 10$ por lo que existe ausencia de multicolinealidad. Además, como se observa en la figura 1 este modelo predictor por medio del Análisis Factorial Confirmatorio (AFC) también muestra un ajuste estructural adecuado dentro de los distintos parámetros estimados.

\section{Discusión}

Los objetivos del estudio comprendieron conocer la relación y la determinación existente entre los indicadores del Bienestar Psicológico y Subjetivo en el Bienestar Laboral en un conjunto de trabajadores de la salud de tres centros de atención sanitaria de la provincia de Bolívar, Ecuador.

Sobre el desempeño del Bienestar Psicológico, Subjetivo y Laboral tanto para médicos como para enfermeras en los centros sanitarios evaluados, de manera general se en- cuentran en un nivel óptimo de percepción y valoración de su satisfacción vital, sus emociones y sus deseos de superación; así como también para la percepción de satisfacción laboral que le genera su trabajo. Resultados similares han sido reportados previamente en personal de salud para el bienestar psicológico y subjetivo (Landa et al., 2006; Oramas, Santana \& Vergara, 2006; Paschoal, Álvaro \& Barreiros-Porto, 2015; Arrogante, Pérez-García \& Aparicio-Zaldívar, 2015). Al igual que para el BL (Estrada-Rodríguez \& Ramírez-Reyes, 2010; Crespo, 2014; Donoso, Demerouti \& Jiménez, 2015; Paris, 2016).

En el caso del BL se puede inferir, que los mecanismos valorativos y de interpretación por medio del Bienestar Psicosocial y los Efectos colaterales al parecer le permiten establecer al trabajador un marco referencial del sentir y la satisfacción que le genera su trabajo. De este modo, el modelo de evaluación de Blanch y otros (2010) encaja dentro del criterio de pluridimensionalidad e integralidad que influyen en el desempeño laboral (Suomaa et al., 2011).

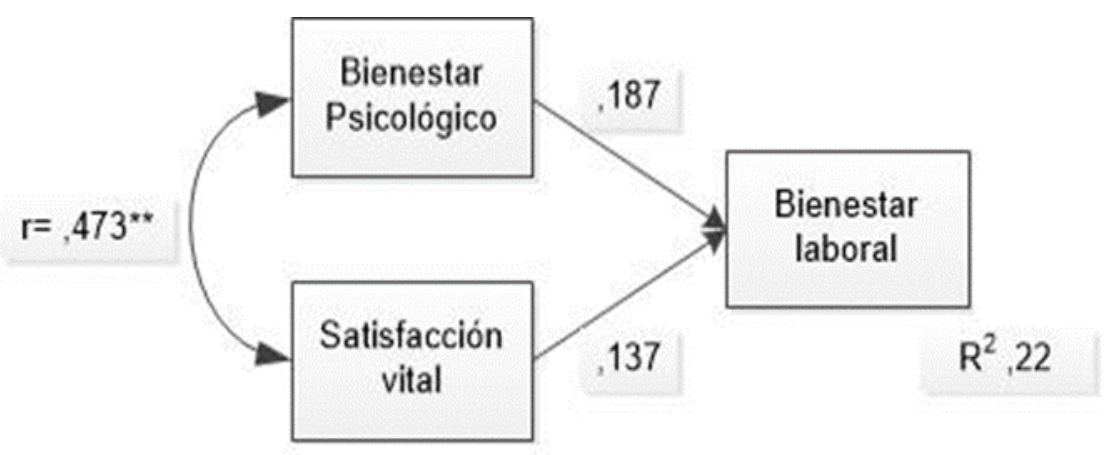

$x^{2}=96,2 ; g l=62 ; p<, 001 ; x^{2} / g l=1,6 ;$ CFI $=, 96 ;$ TLI;, $95 ;$ RMSEA $=, 06[, 04-, 09]$

Figura 1. Modelo de predicción del Bienestar Laboral a partir del

Bienestar Psicológico y la Satisfacción vital 
La presencia considerable del Bienestar evidencia como lo señala Diener, Oishi y Lucas (2003) de la existencia de mecanismos de interpretación favorables del contexto individual y organizacional, para así sentirse cómodo y satisfecho. Esto es hecho precisamente relevante que permite corroborar la importancia que tiene el bienestar para el desarrollo de una actividad laboral y la cultura organizacional (Oswald, Proto \& Sgroi, 2015). Y como previamente sugirieron Nader, Bernate y Santa Bárbara (2014), los hallazgos confirman la mecánica tanto del trabajador cómo de la organización (en especial de instituciones sanitarias) para convertirse en promotoras directas o indirectas de salud organizacional. Aunque esta inferencia requiere estudios confirmatorios a futuro.

Por otra parte, con respecto a los análisis de correlación general, se encontró que efectivamente el Bienestar Psicológico y Subjetivo (satisfacción vital y afecto negativo) muestran relación con el Bienestar Laboral General. Y aunque previamente se encuentran referencias mostrando tal relación (Romeiro Martínez, 2015; Riquena, 2000; Garrosa et al., 2010), no existen análisis sistematizados e integrales del enfoque positivo del bienestar (Ryff, 1989; Seligman, 2003; Diener, Oishi \& Lucas, 2003) en el bienestar laboral, por lo que este estudio es pionero y da un avance significativo en el entendimiento del bienestar a través del trabajo en población ecuatoriana. En esta misma línea de análisis, la relación más representativa del Bienestar Laboral General es con el Bienestar Psicológico. De ahí que se desprende que el esfuerzo por alcanzar metas personales nutre también el sentirse bien en el trabajo y probablemente explica la asociación que guarda con la productividad (Oswald, Proto \& Sgroi, 2015).
La relación entre todos los indicadores también nos señala el potencial predictor del bienestar individual (Psicológico y Subjetivo) en el laboral, pues explica el 22\% de los cambios de varianza. Es decir que, en contradicción a lo mencionado por Florencia (2015) y Restrepo Escobar y López Velásquez (2013), las condiciones laborales no son exclusivas en la formulación del BL, sino que una parte significativa de este constructo, depende de la percepción positiva y satisfacción de sí mismo para sentir satisfacción y felicidad laboral.

Entre las implicaciones de este trabajo se considera gracias a la evidencia mostrada que existe una mejor comprensión de las capacidades personales y organizacionales presentes que permiten el desarrollo del bienestar en el trabajo; como menciona Schaufeli \& Bakker (2004) se aporta en la revisión de los aspectos positivos que ejercen el contexto laboral y organizacional. Y que da muestra de que, si bien el trabajo es causante de desajustes, sobre todo para cumplir con la productividad, también es una herramienta de crecimiento, desarrollo y satisfacción (Nader, Bernate \& Santa-Bárbara, 2014).

\section{Conclusiones e implicaciones}

De manera general, se concluye que el bienestar tanto psicológico como subjetivo $\mathrm{y}$ laboral se encuentran presentes en el personal de salud analizado, además, no presenta diferencial en su constitución entre grupos de profesionales (médicos y enfermeras). De la misma manera tanto el BP, como el BS y el BL se correlacionan entre sí en el tipo de muestra analizada, con mayor fuerza de relación del BP. Así mismo el BP y la Satisfacción con la vida como componente del BS son predictores multifactoriales del BP. Con ello se puede considerar de la relevancia de la percepción del bienestar en la construc- 
ción del BL y la relación con el desempeño laboral.

Las implicaciones prácticas de los hallazgos permiten considerar que las herramientas utilizadas pueden ser instrumentos útiles y pertinentes para procesos de evaluación individuales y organizacionales del bienestar desde un punto de vista positivo e integral. Así como también en el abordaje para la elaboración de planes y procesos de fortalecimiento del bienestar y la salud organizacional positiva en centros sanitarios y otros similares.

\section{Limitaciones y recomendaciones a futuro}

Se debe considerar que una limitación del estudio está en la muestra seleccionada, pues estuvo compuestas por personal de la salud como médicos y enfermeras en centros de atención sanitario, por lo que estos resultados cuentan con bastante limitación en cuanto a representatividad y generalización. Razón por la cual se requieren a futuro nuevos trabajos en el que se muestra mayor heterogeneidad.

\section{Referencias}

Arias, P. R., \& García, F. E. (2018). Propiedades psicométricas de la Escala de Satisfacción con la Vida en población ecuatoriana adulta. Pensamiento Psicológico, 19(2), 21-29. doi:10.11144/javerianacali.ppsi16-2.ppes.

Arrogante, Ó., Pérez-García, A. M., \& AparicioZaldívar, E. G. (2015). Bienestar psicológico en enfermería: relaciones con resiliencia y afrontamiento. Enfermería Clínica, 25(2), 7380. doi:10.1016/j.enfcli.2014.12.009.

Ato, M., López, J., \& Benavente, A. (2013). Un sistema de clasificación de los diseños de investigación en psicología. A nales de Psicología, 29 (3), 1038-1059. doi:10.6018/ analesps.29.3.178511.

Blanch, J. M., Sahagún, M., Cantera, L., \& Cervantes, G. (2010). Cuestionario de bienestar laboral general: estructura y propiedades psicométricas. Revista de Psicología del Trabajo y de las
Organizaciones, 26(2), 157-170. doi:10.5093/ tr2010v26n2a7.

Crespo, J. L. (2014). El bienestar laboral de los profesionales de la medicina: una comparativa iberoamericana. Revista del Centro de Estudios de Sociología del Trabajo, 6, 39-71.

De Cuyper, N., Isaksson, K., \& De Witte, H. (Edits.). (2005). Employment contracts and well-being among European workers. Aldershot: Ashgate.

Díaz, D., Rodríguez-Carvajal, R., Blanco, A., MorenoJiménez, B., Gallardo, I., Valle, C., \& van Dierendonck, D. (2006). Adaptación española de las escalas de bienestar psicológico de Ryff. Psicothema, 18(3), 572-577.

Díaz, D., Stavraki, M., Blanco, A., \& Gandarillas, B. (2015). The eudaimonic component of satisfaction with life and psychological well-being in Spanish cultures. Psicothema, 27(3), 247253.

Diener, E., Emmons, R., Larsen, R., \& Griffin, S. (1985). The Satisfaction With Life Scale. Journal of Personality Assessment, 49(1), 7175. doi:10.1207/s15327752jpa4901_13.

Diener, E., Oishi, S., \& Lucas, R. E. (2003). Personality, culture, and subjective well-being: Emotional and cognitive evaluations of life. Annual Review of Psychology, 54, 403-425. doi:10.1146/ annurev.psych.54.101601.145056.

Donoso, L. M., Demerouti, E., \& Jiménez, B. (2015). Beneficios positivos de cuidar en la motivación y el bienestar del personal de enfermería: un estudio de diario sobre el papel de las habilidades de regulación emocional en el trabajo. International Journal of Nursing Studies, 52, 804-816. https://doi.org/10.12961/ aprl.2015.18.3.08.

Estrada-Rodríguez, Y., \& Ramírez-Reyes, M. (2010). El bienestar laboral y su incidencia en la gestión exitosa de las empresas en el turismo. TURyDES, 3(8), 1-12.

Fierro-Arias, L., Simón-Díaz, P., Ponce-Olivera, R. M., \& Arenas-Guzmán, R. (2018). Estudio sobre la satisfacción laboral y la felicidad en médicos dermatólogos. Gaceta Médica de México, 154(1), 26-35. doi:10.24875/ GMM.17002763.

Florencia, M. (2015). Psicotecnia. Historia de un encuentro entre la psicología y la técnica. Revista Internacional sobre Subjetividad, Pólitica y Arte, 71-85. 
Gaibor-González, I., \& Moreta-Herrera, R. (2020). Optimismo disposicional, ansiedad, depresión y estrés en una muestra del Ecuador. Análisis inter-género y de predicción. Actualidades en Psicología, 34(129), 17-31. doi:10.15517/ AP.V34I129.35148.

Gálvez-Ruiz, P., Grimaldi-Puyana, M., SánchezOliver, A. J., Fernández-Gavira, J., \& GarcíaFernández, J. (2017). Cultura organizacional y satisfacción laboral: Propiedades psicométricas de una escala en centros de fitness. Revista de psicología del deporte, 26(Suppl. 4), 104 -109 .

García, F. E., Cova-Solar, F., Bustos-Torres, F., \& Reyes-Pérez, É. (2018). Burnout y bienestar psicológico de funcionarios de unidades de cuidados intensivos. Duazary, 15(1), 23-38. doi:10.21676/2389783X.2101.

Garrosa, E., Moreno, B., Boada, M., \& Blanco, L. M. (2010). Emociones positivas y bienestar laboral. Gestión práctica de riesgos laborales: Integración y desarrollo de la gestión de la prevención, 74, 22-27.

González, P., \& Bousoño, M. (1996). Evaluación de calidad de vida. Psiquiatría, V(6), 47-54.

IBM Corp. (2012). IBM SPSS Statistics for Windows. Armonk, NY: IBM Corp.

Joiner, T., Sandin, V., Chorot, P., \& Marquina, L. (1997). Development and Factor Analytic Validation of the SPANAS Among Women in Spain. Journal of Personality Assessment, 68 (3), 600-615. doi:10.1207/ s15327752jpa6803_8.

Landa, J. M., Berrios-Martos, M. P., López-Zafra, E., \& Aguilar Luzón, M. (2006). Relación entre Burnout e Inteligencia emocional y su impacto en Salud mental, Bienestar y Satisfacción Laboral en profesionales de enfermería. $A n$ siedad y estrés, 12(2-3), 479-493.

Medrano, L. A., Kanter, E. F., Trógolo, M., Ríos, M., Curerello, A., \& González, J. (2015). Adaptación de la Escala de Afecto Positivo y Negativo (PANAS) para la población de Estudiantes Universitarios de Córdoba. Anuario de Investigaciones de la Facultad de Psicología, 2(1), 22-36.

Moreta, R., Gaibor, I., \& Barrera, L. (2017). El bienestar psicológico y la satisfacción con la vida como predictores del bienestar social en una muestra de universitarios ecuatorianos. Salud \& Sociedad, 8(2), 172-184. doi:/10.22199/ s07187475.2017.0002.00005.

Moreta-Herrera, R., López-Calle, C., Gordón-Villalva, P., Ortíz-Ochoa, W., \& Gaibor-González, I.
(2018). Satisfacción con la vida, Bienestar Psicológico y Social como predictores de la salud mental en ecuatorianos. Actualidades en Psicología, 32(124), 111-125. doi:10.15517/ ap.v32i124.31989.

Nader, M., Bernate, S. P., \& Santa-Bárbara, E. S. (2014). Predicción de la satisfacción y el bienestar en el trabajo: hacia un modelo de organización saludable en Colombia. Estudios Gerenciales, 30(130), 31-39. doi:/10.1016/ j.estger.2014.02.006.

OIT. (2016). Estrés en el trabajo: Un reto colectivo. Ginebra: OIT.

Oramas, A., Santana, S., \& Vergara, A. (2006). El bienestar psicológico, un indicador positivo de la salud mental. Revista Cubana de Salud y Trabajo, 7(1-2), 34-39.

Organización Mundial de la Salud [OMS]. (2013). Organización Mundial de la Salud. Obtenido de Salud mental: un estado de bienestar: http://www.who.int/features/factfiles/ mental_health/es/

Oswald, A. J., Proto, E., \& Sgroi, D. (2015). Happiness and productivity. Journal of Labor Economics, 33(4), 789-822. doi:.1086/681096.

Padrós, F., Gutiérrez, C., \& Medina, M. (2015). Propiedades psicométricas de la Escala de Satisfacción con la Vida (SWLS) de Diener en población de Michoacán (México). Avances en Psicología Latinoamericana, 33(2), 221230. doi:10.12804/apl33.02.2015.04.

Paris, L. (2016). Estrategias de afrontamiento del estrés asistencial y satisfacción laboral-personal en médicos y enfermeros. Revista interamericana de Psicología ocupacional, 26(2), 7-21.

Paschoal, T., Álvaro, J. L., \& Barreiros-Porto, J. (2015). El efecto moderador de los valores personales en la relación entre condiciones de trabajo y bienestar. Revista de Psicología Social, $\quad 30(1), \quad 89-121$. doi:.1080/02134748.2014.987505.

Peiró, J., Tordera, N., Lorente, L., Rodríguez, I., Ayala, Y., \& Latorre, F. (2014). Bienestar sostenible en el trabajo. Conceptualización, antecedentes y retos. PSIENCIA: Revista Latinoamericana de Ciencia Psicológica, 7(1), 133135.

Peña-Garzón, K., \& Torrente-Rocha, J. (2020). Aprendizaje organizacional e innovación en las tiendas de cafés especiales de Ibagué, Colombia. Veritas \& Research, 2(1), 49-59.

Ravenswood, K. (2011). Productivity, Participation and Employee Wellbeing in the Residential 
Aged Care Sector (Unpublished PhD Thesis). AUT University, New Zealand: AUT University.

Requena, F. (2000). Satisfacción, bienestar y calidad de vida en el trabajo. Revista Española de Investigaciones Sociológicas(92), 11-44. doi:10.2307/40184292.

Restrepo Escobar, F., \& López Velásquez, A. (2013). Percepciones del entorno laboral de los profesionales universitarios en un contexto de reorganización flexible del trabajo. Cuadernos de Administración, $\quad 29(49), \quad 55 \quad-\quad 63$. doi:10.25100/cdea.v29i49.64.

Romeiro Martínez, S. (2015). Bienestar psicológico y laboral en los docentes: un estudio empírico correlacional. A randu-UTIC. Revista Cientifica Internacional de la Universidad Tecnológica Intercontinental, 2(1), 123-148.

Ryff, C. (1989). Beyond Ponce de Leon and life satisfaction: New directions in quest of successful ageing. International Journal of Behavioral Development, $\quad 12, \quad 35-55$. doi:10.1177/016502548901200102.

Schaufeli, W. B., \& Bakker, A. B. (2004). Job demands, job resources, and their relationship with burnout and engagement: A multisample study. Journal of organizational Behavior, 25(3), 293-315 doi:10.1002/job.248.

Seligman, M. E. (2003). La auténtica felicidad. Barcelona: Vergara.

Suomaa, L., Yrjänheikki, E., Savolainen, H., \& Jokiluoma, H. (2011). 5. Excellence in risk assessment and well-being at work. Healthy workplaces, 12, 22-23.

Torres-Salazar, C., Moreta-Herrera, R., RamosRamírez, M., \& López-Castro, J. (2020). Sesgos Cognitivo de Optimismo y Percepción de Bienestar en una Muestra de Universitarios Ecuatorianos. Revista Colombiana de Psicología, 29(1), 61-72. doi:10.15446/ rcp.v29n1.75853.

Van Aerden, K., Moors, G., Levecque, K., \& Vanroelen, C. (2015). The relationship between employment quality and work-related well-being in the European Labor Force. Journal of $\mathrm{Vo}-$ cational Behavior, 86, 66-76. doi:10.1080/16184742.2017.1406971.

van Dierendonck, D. (2004). The construct validity of Ryff's Scale of Psychological well-being and its extension with spiritual well-being. Personality and Individual Differences, 36(3), 629-644. doi:10.1016/s0191-8869(03)001223.

Warr, P. B. (2002). Psychology at work (5ta ed.). Londres: Penguin Books.
Watson, D. (1988). Intraindividual and interindividual analyses of positive and negative affect: Their relation to health complaints, perceived stress, and daily activities. Journal of Personality and Social Psychology, 54, 1020-1030. doi:10.1037//0022-3514.54.6.1020. 